\title{
Getting the right patient to angiography: Can we level the playing field?
}

\author{
P. S. Jayadeva, ${ }^{a}$ and N. Better ${ }^{a, b, c}$ \\ a Department of Cardiology, Royal Melbourne Hospital, Parkville, Melbourne, Australia \\ b Department of Nuclear Medicine, Royal Melbourne Hospital, Parkville, Melbourne, Australia \\ c University of Melbourne, Parkville, Melbourne, Australia
}

Received Nov 25, 2020; accepted Nov 28, 2020

doi: $10.1007 / \mathrm{s} 12350-020-02475-9$

\section{See related article, pp. 1141-1155}

The weight of non-traditional cardiovascular (CV) risk factors and their role in influencing cardiac outcomes in general has been previously described. ${ }^{1}$ Gowdar et al. have explored the role of these risk factors and their influence in referral for invasive coronary angiography following myocardial perfusion imaging (MPI). Non-traditional risk factors explored included race/ethnicity, socioeconomic factors, marital and insurance status.

A decision to proceed to invasive coronary angiography based on traditional $\mathrm{CV}$ risk factors to alter prognosis, improve symptoms and change outcomes has been explored by many previous studies. The weight of traditional cardiovascular factors plays a vital role not only in referral to angiography but also in the referral for provocative testing in the first place. The management of demonstrable ischemia in "stable" patients has been widely debated and an initial medical approach is not unreasonable, even in the setting of a moderate to large amount of ischemia, as demonstrated in the ISCHEMIA trial. ${ }^{2}$ The weight of accumulating traditional risk factors tips the scale to favour provocative testing in high risk patients but may not necessarily lead to adverse outcomes if guideline directed optimal medical therapy alone is initiated once ischemia is defined. ${ }^{2}$

Reprint requests: N. Better, Department of Cardiology, Royal Melbourne Hospital, Parkville, Melbourne, Australia; nathan.better@mh.org.au

J Nucl Cardiol 2022;29:1156-8.

$1071-3581 / \$ 34.00$

Copyright (c) 2021 American Society of Nuclear Cardiology.
27,895 patients were included in Gowdar's retrospective study; the standout result was Caucasian ethnicity and private or Medicare insurance status being associated with referral to angiography, even after controlling for traditional risk factors. These non-traditional risk factors lead to coronary angiography referral even when taking into account the severity of the MPI findings (i.e. these disparities persisted even in patients with moderate or severely abnormal MPIs). Hispanic patients were the least likely to undergo angiography when compared to their Caucasian counterparts. Previous studies have highlighted African American patients being less likely to be referred for coronary angiography; however, in this study the differences in referral to angiography in this cohort was not statistically significant. Similar findings were mirrored in previous trials where minority ethnicity groups such as individuals of African American and Hispanic origin were less likely to be referred for coronary revascularisation and less likely to be hospitalised for an acute coronary syndrome (ACS), sometimes despite the presence of significant coronary artery disease (CAD). ${ }^{3-5}$

Are the rates of referral for invasive coronary assessment lower for African American and Hispanic patients because the same bias and disadvantage applies to their pre hospitalised care as it does for their in-hospital management? How do we mitigate the disparities in $\mathrm{CV}$ risk management and downstream investigation of coronary artery disease in these minority groups? The literature has previously described minority groups differing in the management of CV risk factors and having a greater rates of morbidity and mortality associated with acute coronary syndromes. ${ }^{1}$

The evidence for racial disparity has been extensively investigated, especially with use of diagnostic cardiovascular procedures and coronary revascularization. $^{6}$ Lurie et al explored the behaviour of 344 
cardiologists and their awareness of these disparities. $33 \%$ agreed to the existence of these disparities in cardiovascular care, which is not a substantial proportion, and $58 \%$ agreed that care also differed according to insurance status. ${ }^{6}$ First and foremost there seems to be a lack of awareness of these disparities, inversely proportional to the proximity to their own practice setting, which allows factors such as ethnicity and insurance status to be pervasive, either consciously or subconsciously in the decision making pathway of patients presenting with cardiac symptoms. These disparities seem to be present from time of initial consultation to the cardiac catheterisation laboratory.

Insurance status has also emerged as a factor in influencing referral to angiography with privately insured patients and Medicare patients more likely to be invasively investigated. Previous observational studies have described greater traditional $\mathrm{CV}$ risk factor presence in uninsured patients and as a consequence, poorer outcomes were observed. ${ }^{7,8}$ Insurance status can be an extension of socioeconomic status. Those who are privately insured and Medicare covered may have had a greater referral to coronary angiography, simply due to the cohort being able to afford these services. Are those without insurance or Medicare medically managed more aggressively? Or are invasive procedures favoured in those that can afford them or perceived favourably by patients in this group; patient preference and attitude also influences such decision making. The downstream consequence of angiography referral is hospital stay, further addition of costs if percutaneous coronary intervention is performed or greater length of hospital stay for patients requiring surgical revascularisation. This is more palatable in patients covered with insurance or Medicare.

Interestingly, 454 normal MPI studies (1.6\%) underwent coronary angiography and $12.2 \%$ of patients with an abnormal MPI did not undergo coronary angiography. Patients that underwent coronary angiography did tend to have more abnormal MPI results (moderate to severely abnormal) and ECG changes during stress was highlighted as an important factor in the push towards invasive management along with an abnormal MPI. The reason for invasive assessment of patients with normal MPI can be determined by varying factors. Additional cardiac factors such as left ventricular (LV) dysfunction, the presence of coronary calcium, traditional or non-traditional risk factors or simply persistent symptoms may further influence clinician referral for angiography. This may be to obtain a "definitive diagnosis" or to further risk stratify for prognosis (e.g. the presence of increasing coronary calcium is associated with adverse cardiovascular outcomes ${ }^{9}$ ). This study does not explore the rates of revascularization in each MPI group (mild/moderate/severe perfusion defects) or whether the cohort of patients with abnormal or normal MPIs were referred for additional anatomical testing such as a CT coronary angiography (CTCA). A large percentage of the patients studied, were prior to the CTCA era.

Whilst traditional $\mathrm{CV}$ risk factors are tangible contributors to $\mathrm{CV}$ risk, factors such as race/ethnicity, insurance status and the bias they inflict on the referring and assessing clinician is more difficult to predict. Partly, other factors such as the attitude to invasive testing held by patient groups and level of education, which was not directly measured in this study, may also have an influence. ${ }^{1}$ Bias can potentially exist at many points along the diagnostic and management pathway of patients with cardiac symptoms and risk factors. This bias may exist in the patient and their willingness to undergo testing, in the primary care physician who often knows the patient best or in the treating cardiologist. In this study, all patients were managed by a treating cardiologist. Previously, minority groups, in particular African American patients were less likely to be referred to a cardiologist in the first place and thus undergo coronary angiography, despite having a $30 \%$ greater likelihood of dying from heart disease when compared to Caucasian individuals (non-white/non-Hispanics). ${ }^{1}$

An extrapolation from this study is the implication of non-traditional risk factors and their burden in global $\mathrm{CV}$ disease and mortality. Although the health infrastructure varies between countries, the varying population distribution and insurance issues offer parallels to Gowdar's work. Access and thus implementation of nuclear cardiac imaging in developing countries is limited. ${ }^{10}$ Given the reduced referral of non-Caucasian patients to invasive cardiac investigation, and noting that an abnormal MPI was the strongest predictor of referral to angiography, the implication in the developing world may be critical. If access to coronary angiography is also limited, often for financial reasons, identifying patients that need revascularization becomes increasingly important, and should ideally extend beyond those who can afford it or insurance status. Hence, the initial demonstration of ischemia to guide medical therapy or angiography may potentially offer a cost-effective strategy to allow such selection, making the argument for promoting access to nuclear imaging in these countries even stronger. Of course, this argument is theoretical, but whets the appetite as to how the traditional and non-traditional risk factors can intertwine in different population settings. 
The authors should be commended on their work in exploring the question of non-traditional $\mathrm{CV}$ risk factors influencing coronary angiography referral, which ultimately impacts on downstream and future cardiac management in high risk cohorts. The population was substantial in nature, and although limited by the retrospective nature of the study, the reader should now be aware of considering other factors, rightly or wrongly, besides the MPI result in determining referral to angiography. Ideally, prospective studies evaluating long-term outcomes in these different cohorts may provide answers, but would be difficult to undertake.

Whilst MPI is a gatekeeper in patient selection for invasive coronary assessment and assisting to define and target coronary revascularization, non-traditional risk factors and their influence on referral, as explored by Gowdar et al, has potential implications to skew referral for angiography. Ultimately the decision to proceed with angiography rests with both the clinician and patient; however, the influence of healthcare infrastructure and health behaviour should not be taken for granted. Despite advances in cardiovascular risk management and intervention, minority populations have not observed the same decline in mortality and morbidity from CV disease as Caucasian patient cohorts. In order to level the playing field and facilitate timely referral to angiography, active awareness of these non-traditional risk factors by as outlined by Gowder et al. is key.

\section{References}

1. Graham G (2015) Disparities in cardiovascular disease risk in the United States. Curr Cardiol Rev 11:238-45

2. Maron DJ et al (2020) Initial invasive or conservative strategy for stable coronary disease. N Engl J Med 382:1395-07

3. Barnhart JM, Wassertheil-Smoller S, Monrad ES (2000) Clinical and nonclinical correlates of racial and ethnic differences in recommendation patterns for coronary revascularization. Clin Cardiol 23:580-86

4. Singh JA et al (2014) Trends in and disparities for acute myocardial infarction: An analysis of Medicare claims data from 1992 to 2010. BMC Med 12:190

5. Philbin EF et al (2000) Socioeconomic status is an important determinant of the use of invasive procedures after acute myocardial infarction in New York State. Circulation 102:107-15

6. Lurie $\mathrm{N}$ et al (2005) Racial and ethnic disparities in care: The perspectives of cardiologists. Circulation 111:1264-69

7. Fowler-Brown A et al (2007) Risk of cardiovascular events and death-does insurance matter? J Gen Intern Med 22:502-07

8. $\mathrm{Ng} \mathrm{DK}$ et al (2012) Insurance status, not race, is associated with mortality after an acute cardiovascular event in Maryland. J Gen Intern Med 27:1368-76

9. Engbers EM et al (2016) Prognostic value of coronary artery calcium scoring in addition to single-photon emission computed tomographic myocardial perfusion imaging in symptomatic patients. Circ Cardiovasc Imaging 9:e003966

10. Vitola JV (2016) Nuclear cardiology and CVD in the developing world: Are we applying our scarce resources appropriately? Why is our mortality rate so high? J Nucl Cardiol 23:1166-70

Publisher's Note Springer Nature remains neutral with regard to jurisdictional claims in published maps and institutional affiliations. 